\title{
Design and Implementation of Remote Anonymous Attestation Protocol Based on Trusted Cloud Computing Platform
}

\author{
Liu Yan ${ }^{1, *}$ and Xia Bin $^{2}$ \\ ${ }^{I}$ College of Information Engineering, Zhongzhou University, Zhengzhou, 450044, P.R. China; ${ }^{2}$ School of Information \\ and Management Science, Henan Agricultural University, Zhengzhou, 450002, P.R. China
}

\begin{abstract}
Trusted cloud computing platform is a combination of the use of virtual machine technology and trusted computing technology of cloud computing platform. Virtual machine technology because of its high separability of the client and the resource of high controllability, which greatly improves the security of the system; and the trusted computing technology is through the establishment of a trusted root in the hardware layer, solve the credibility and safety problem of the system fundamentally, because of the close combination of Trusted Computing and the virtual machine technology to establish a "trusted cloud computing" can ensure that the user data and application in cloud environment fundamentally safe. In this paper, the trusted computing technology and properties of signature mechanism based on the proposed a trusted cloud is simple, safe and efficient computing platform remote anonymous attestation protocol RAA-TCCP protocol. The protocols without bilinear pairings, also need not attribute certificate and AIK certificate, which greatly simplifies the certificate management, and simultaneously realize the identity and integrity of the state of computing nodes to prove. The protocol has unforgeability, platform identity anonymity, privacy protection and allocation of collusion resistance, also has the very high efficiency even in a high security strength, good to meet the safety requirements and performance requirements of the cloud environment, remote attestation.
\end{abstract}

Keywords: Protocol, remote attestation, trusted cloud computing, Virtual machine technology.

\section{INTRODUCTION}

Trusted cloud computing platform is a combination of the use of virtual machine technology and trusted computing technology of cloud computing platform. Virtual machine technology because of its high separability of the client and the resource of high controllability, which greatly improves the security of the system; and the trusted computing technology is through the establishment of a trusted root in the hardware layer, solve the credibility and safety problem of the system fundamentally, because of the close combination of Trusted Computing and the virtual machine technology and the establishment of a "trusted cloud computing", can ensure the user data and application in cloud environment fundamentally safe.

At present, the trusted platform remote attestation mainly has two forms: proof of identity for the platform and platform for state integrity proof. Platform for proof of identity the typical Privacy based CA (Privacy Certificate Authorities [1]) proof, proof of based ring signature [2, 3]. Proof based on ring signature is a kind of more effective remote attestation scheme, it will ring signature is hidden in the ring members, to avoid the leakage of TPM platform identity, but a number of ring members determines the length of the ring signature and the anonymity, efficiency, by reducing the number of ring members reduce ring signature length and Improve the efficiency of the signature will sacrifice anonymity for the price. And according to the platform integrity state proved that the typical TCG binary proof (Binary Attestation), based on the attributes of the proof of (Property-based Attestation, PBA) etc. The TCG binary proved to be the most basic method of remote attestation; Remote Attestation Based on attribute to overcome the existing remote attestation complexity, privacy leakage and abuse of proven results and other defects. However, these remote attestation methods have low efficiency, certificate management difficulty is big, can not resist collusion attack and other issues, practicality is not high. For cloud computing platform, due to the uncertainty, mobility and opacity of compute nodes, these methods are more difficult to use. So it is urgent to an efficient, anonymous remote attestation scheme to solve the problem of cloud computing platform to prove the credibility. To this end, in 2009, Santos proposed a trusted cloud computing platform (Trusted Cloud Computing Platform, TCCP) model, turned to address cloud computing platform credibility ideas proved problem using trusted computing technology [4].

This paper assumes that the hardware resources is credible, combining with binary certificate and attribute based ring signature to prove, using the idea, design a simple, efficient cloud computing platform remote anonymous attestation protocol. The protocols without bilinear pairings, also need not attribute certificate and AIK certificate, it is through the use of an off-line trusted third party, application of trusted computing binding and sealing mechanism and attribute based ring signature (Property-Based Ring Signature, PBRS [5]) mechanism, and realizes the identity and integrity of the state of computing nodes to prove, and can not be and 
collusion resistance forgery, identity anonymity, privacy protection configuration. In this paper, called RAA-TCCP (Remote anonymous authentication of trusted cloud computing platform) protocol.

\section{PROTOCOL DESCRIPTION}

In this paper, RAA-TCCP protocol from the abstract of a group, the platform configuration register (PCRs) in binary metric value into one or more attribute values, and with the platform, and then on the basis of attribute based ring signature (PBRS), credibility by verifying the ring signed the legitimacy to judge the computing nodes the. PBRS is using a ring signature concept attribute based signature. It will have the same attribute members form a ring, ring members can be with the one or more attributes as the identity. Because of the number of members with the same attributes of the unknown, so the identity of the signer can be hidden in the ring, and he takes a certain property or some attributes as the identity release news, but also can make the verifier believe the authenticity of the message. PBRS has the advantage of flexible, intuitive, and ring signature length and number of ring members independently, so PBRS can be conveniently and efficiently applied to anonymous authentication system.

The dependability of RAA-TCCP protocol suitable for IaaS, PaaS, SaaS and so on the different levels of service to prove. Without loss of generality, to provide the IaaS service as an example to describe the RAA-TCCP protocol. The RAA-TCCP protocol is divided into two phases of proof preparation and proof implementation as shown in Fig. (1). In the proof of the preparation stage, the compute nodes based on the $\mathrm{N}$ have EK certificate and PCR set of metrics signature key to a trusted third party TC application, TC binary verify its trusted after given its attribute value based on the sign and seal and signature key. In the proof of the implementation stage, the compute nodes $\mathrm{N}$ ring signature using the signature key, prove its security properties of the corresponding, user $U$ and then through the validation of ring signature to determine the credibility of $\mathrm{N}$, and then will be entrusted to the virtual machine to the trusted computing nodes. In order to facilitate the description, the following definitions symbol: $E K_{A}^{P}$ shows the $E K$ public key of $A$; $P_{A}$ shows $A$ generated public key; $\{X\}_{K}$ shows $X$ is generated by key $K$ ciphertext; $K_{A B}$ shows between $A$ and $B$ session key; $A \rightarrow B$ : $X$ shows $A$ sent message $X$ to $B ; n_{A}$ shows nonce by $A$ to prevent replay attacks; TPM $A$ shows a trusted platform module $A ; V_{P C R-A}$ shows a group $P C R G_{P C R-A}$ value of $T P M_{A}$.

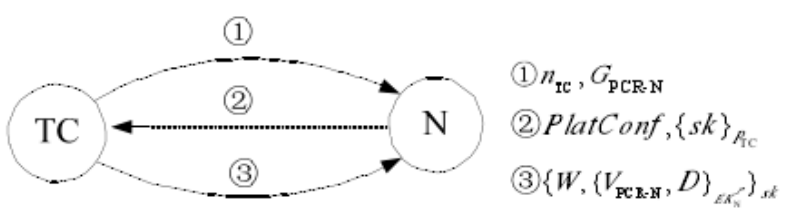

a) Proof Preparation

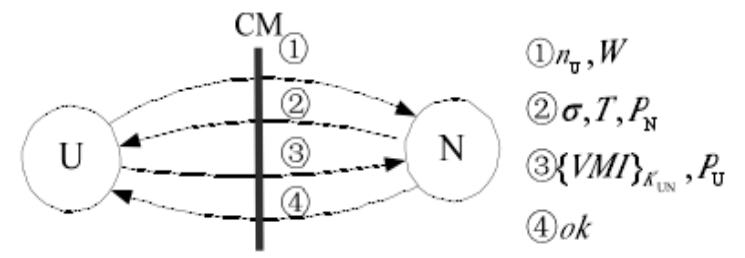

b) Proof Implementation

Fig. (1). RAA-TCCP protocol.

\subsection{Proof Preparation}

(1) System Established

Let $E$ be the elliptic curve over a field $F_{q}, q$ is a prime number, $E\left(F_{q}\right)$ is the set of all points of $E,{ }_{\#} E\left(F_{q}\right)$ is factorial of $E, P \in E\left(F_{q}\right)$ is the generating element elliptic curve of group $\langle p\rangle$, the order for a divisible ${ }_{\#} E\left(F_{q}\right)$ the big prime number $p$. TC randomly selects a private key $x \in Z_{p}^{*}$ and the two has the following form of collision free one-way Hash function: $H_{0}:\{0,1\}^{*} \rightarrow\{0,1\}^{K}, H_{1}:\{0,1\}^{*} \rightarrow Z_{p}^{*}$. where $\{0,1\}^{K}$ is the session key space. The complete definition of attribute value is $Z_{p}$. Remember x coordinates of $Q \in\langle P\rangle$ is $x_{Q}=[Q]_{x}$.

TC public $p, P, H_{0}, H_{1}$ and public key $P_{T C}=x P$, secure private key $x$.

(2) Attribute Certification

TC first $\mathrm{N}$ certification which has given security attributes, and attribute value. Because the property is an abstract concept, how will the property abstract information into the concrete numerical method based on attribute is the key. In this paper, the attribute abstraction method as shown in Fig. (2).

Definition 1: Attribute abstract is a function solving process from the PCR set of metrics to the attribute values as shown in Fig. (2), figure $\stackrel{m n}{f_{i}}$ shows mapping relation of $f_{i(i=1,2,3,4,5,6)}$, on which the digital representation of the

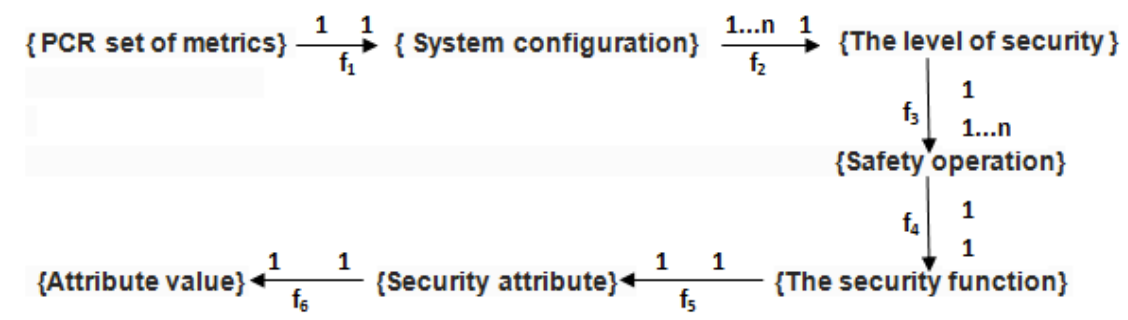

Fig. (2). Attribute abstraction method. 
mapping types: $f_{1}, f_{4}, f_{5}$ and $f_{6}$ is a one to one mapping, $f_{2}$ is a many to one mapping, $f_{3}$ is the one to many mapping. This process is denoted as $F=F \circ f_{1}$, wherein, $f_{1}$ is called to verify the configuration function, its value is determined by SML, $F=f_{6} \circ f_{5} \circ f_{4} \circ f_{3} \circ f_{2}$ is called the attribute verification function, its value is determined by the security attribute evaluation criteria.

Note GPCRVALS represents a collection of $\{$ PCR set of metrics\}, PROPVALS represents a collection of \{attribute values $\}$, it is not difficult to infer from Fig. (2), function $F: G P C R V A L S \rightarrow P R O P V A L S$ is a many to many mapping. This function to PCR set of metrics Abstract property, because the PCR value represents the platform configuration state, and reflects the system components to start the order, so that the abstract is more simple and accurate properties.

Based on the function of $\mathrm{F}$, the $\mathrm{N}$ property of the certification procedure is as follows:

$$
T C \rightarrow N: n_{T C}, G_{P C R-N}
$$

TC sends challenge $n_{T C}$ to $\mathrm{N}$, request $\mathrm{N}$ provides $G_{P C R-N}$ of PCR group, as shown in Fig. (1a).

$$
N \rightarrow T C: \text { PlatConf },\{S K\}_{P_{T C}}
$$

$\mathrm{N}$ interacts with TSS, request $T P M_{N}$ to generate a session key generated $s k$, generate $\{S K\}_{P_{T C}}$ and PlatConf $=$ $\left\{V_{P C R-N}, n_{T C}, S M L, \text { EndCred, PlatCred,ConCred }\right\}_{s k}$, and then send it to the TC, as shown in Fig. (1a) (2). Among them, $S M L$ is the metric $\log$ of the corresponding to $V_{P C R-N}$; EndCred is a certificate for EK (Endorsement Credential), is used to prove TPM manufacturer identity certificate; PlatCred is a platform Certificate, is used to prove the platform manufacturer identity; ConCred is a Conformance Credential, is used to prove TPM as a trusted building blocks (TBB) parts one of its design and implementation, consistent with the evaluation criteria.

The TC message is received after the verification certificate, is correct, whether the message is reproduced. If the verification is successful read SML and assembly standard value, imitation PCR extended operation, get the PCR desired set of metric values and standard PCR set of metrics. If the two is equal to $V_{P C R-N}, \mathrm{~N}$ is proved credible and configuring security, so the calculation of TC value of the $\mathrm{N}$ attribute $F\left(V_{P C R-N}\right)$, and then enter the key issue stage; or otherwise.

(3) Key Issue

Assuming that $\mathrm{N}$ has a security attribute set $W=\left\{\omega_{i}\right\}_{1 \leq i \leq n}=F\left(V_{P C R-N}\right)\left(\omega_{i} \in Z_{P}\right)$, TC issued for the key steps are as follows:
1. Select a random number $r \in Z_{P}{ }^{*}$, calculate $\mathrm{G}=\mathrm{rP}$, $\mathrm{e}=H_{1}(W) \bmod p, \mathrm{t}=(x e+r) \bmod p$, output signature key $\mathrm{D}=(\mathrm{G}, \mathrm{t})$.

2. The TC obtains the $E K_{N}{ }^{P}$ from the EK certificate, binding and sealing for the formation of $\mathrm{D}$, then create $\left\{V_{P C R-N}, D\right\}_{E K_{N}{ }^{P}}$. This D can only be decrypted by $T P M_{N}$, and only when the value $G_{P C R-N}$ equal to $V_{P C R-N}$, $\mathrm{D}$ can be released.

3. The TC generated $\left\{W\left\{V_{P C R-N}, D\right\}_{E K^{P}{ }_{N}}\right\}_{S K}$, and send it to $\mathrm{N}$, then $\mathrm{N}$ saves it, as shown in Fig. (1a).

\subsection{Proof Implementation}

After $\mathrm{N}$ obtained the signature key, can prove its corresponding security properties set to the user $\mathrm{U}$, as shown in Fig. (3b). The steps are as follows:

$U \rightarrow N: n_{U}, W$

$\mathrm{U}$ sends to the $\mathrm{N}$ challenge $n_{U}$, request $\mathrm{N}$ proves to have property set $W$.

$N \rightarrow U: \sigma, T, P_{N}$

$\mathrm{N}$ components ring as $W$, generating property signature $\sigma$. First of all, $\mathrm{N}$ interactive TSS, loading $\left\{V_{P C R-N}, D\right\}_{E K_{N}{ }^{P}}$ to $T P M_{N} . T P M_{N}$ checks that if $V_{P C R-N}$ equal to $G_{P C R-N}$. If equal, $T P M_{N}$ provides $D=(\mathrm{G}, \mathrm{t})$, or otherwise. If $D$ is open, $s, r^{\prime} \in Z_{P}^{*}$ were randomly selected by $T P M_{N}$, and calculate $P_{N}=\mathrm{sP}, \quad m=H_{1}\left(n_{u}, W, P_{N}\right), \quad Q=r^{\prime}(P+G) \quad(Q \neq G)$, $\sigma_{1}=\left(m+x_{Q}\right) \bmod \mathrm{p}, \sigma_{2}=\left(r^{\prime}-t \sigma_{1}\right) \bmod \mathrm{p}, T=\left(\sigma_{1}+r^{\prime}\right) G$, generate property signature $\sigma=\left(\sigma_{1}, \sigma_{2}\right)$. N return $\sigma$ together with T, $P_{N}$ to $\mathrm{U}$.

$$
U \rightarrow N:\{V M I\}_{K_{U N}}, P_{U}
$$

After $U$ received the message (2), the calculate $e=H_{1}(W) \bmod p, \quad m=H_{1}\left(n_{U}, W, P_{N}\right)$, $x_{Q^{\prime}}=\left[\sigma_{2} P+e \sigma_{1} P_{T C}+T\right]_{X}, \quad \sigma_{1}^{\prime}=\left(m+X_{Q}\right) \bmod p$, to verify the equation $\sigma_{1}^{\prime}=\sigma_{1}$ (1) Whether to set up. If established, then the $N$ signature key issued by $T C$ and is sealed, so $N$ is trusted, then $U$ select the random number $K \in Z_{P}^{*}$, $P_{U}=k P, K_{U N}=H_{0}\left(k P_{N}\right)$ calculation, and then generate $\{V M I\}_{K_{U N}}$, together with $P_{U}$ sent to the $N$, let the virtual machine image $V M I$ loaded on $N$ start or otherwise;

$$
N \rightarrow U: o k
$$


After $\mathrm{N}$ received message (3), interact with the TSS, $T P M_{N}$ compute $K_{N U}=H_{0}\left(s P_{U}\right)$, obviously $K_{N U}=K_{U N}$, and then decrypt $\{V M I\}_{K_{U N}}$. So $N$ can start the user virtual machine, returns a successful information $o k$

Then, if the user virtual machine needs from the source node to the destination node $A$ transplantation $B$, a virtual machine migration message exchange, as shown in Fig. (3).

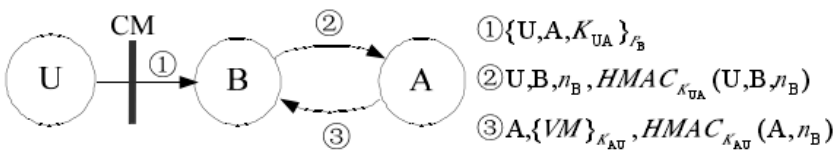

Fig. (3). Virtual machine migration message exchange.

1. $U$ first use the method to validate the $B$ credible, then generate $\left\{U, A, K_{U A}\right\}_{P_{B}}$ sent to the $B$;

2. The $B$ to $K_{U A}$ of $(U, B, n B)$ generates the hash message authentication code $H_{M A C_{K_{U A}}}(U, B, n B)$ is sent to the $A$.

3. $A$ was $K_{A U}$ verified by $H M A C_{K_{A U}}(U, B, n B)$ as legitimate, then the virtual machine of $\mathrm{U}$ users use $K_{A U}$ encryption to send to $B$. Thus to ensure user virtual machine running confidentiality and controllable.

\section{PROTOCOL ANALYSIS}

\subsection{Correctness}

Theorem (1) When attribute signature $\sigma$ is true, equation is correct.

Proof: when $\sigma$ true, correctness of equation (1) is as follows:

$$
\begin{aligned}
& x_{Q^{\prime}}=\left[\sigma_{2} P+e \sigma_{1} P_{T C}+T\right]_{x} \\
& =\left[\sigma_{2} P+e \sigma_{1} P_{T C}+\sigma_{1} G+r^{\prime} G\right]_{x} \\
& =\left[\sigma_{2} P+\sigma_{1}(x e+r) P+r^{\prime} G\right]_{x} \\
& =\left[\left(\sigma_{2}+\sigma_{1} t\right) P+r^{\prime} G\right]_{x} \\
& =\left[r^{\prime} P+r^{\prime} G\right]_{x}=x_{Q}
\end{aligned}
$$

So $\sigma_{1}^{\prime}=\left(m+x_{Q}\right) \bmod p=\left(m+x_{Q}\right) \bmod p=\sigma_{1}$.

\subsection{Safety}

Theorem (2) Under assumption of $D L P$ problem, the attribute signature $\sigma$ is unforgeable. Accurate to say, if the attacker can $A$ with non negligible probability of forging property signature $\sigma^{*}$, then there exists a probability of a $C$ attack algorithm can use the $A$ to solve the $D L P$ can not be ignored. $\mathrm{p}$
Proof: assume that the attacker $A$ can forge property signature $\sigma^{*}$ point at certain platform attributes set $W^{*}, C$ in order to solve the $D L P$, first set the public parameters of paramas: $P, P_{T C}=x P$, and then paramas told the attacker $A$, and kept secret private key $x$. In order to realize the consistency of Oracle queries of the attacker $A, C$ maintain the following lists: $L_{H_{1}}$ is used to store the Hash Oracle $H_{1}$ query and response data; $L_{1}$ is used to store the key of the simulation process of Oracle Issue query and response data; $L_{s}$ is used to store the property proved that the simulating process in Oracle Attest query and response data.

Hash simulation: Set $A$ oracle $H_{1}$ asked to attribute set $W$, if the previous to ask, then return the same result, otherwise $C$ randomly selected $h \in Z_{P}^{*}, H_{1}(w)=h$, answer, and $(W, h)$ is added to the $L_{H_{1}}$.

The key issue of simulation: Set $A$ to the set of attributes of $W\left(W \neq W^{*}\right)$ Oracle Issue asked if the previous to ask, then return the same result, otherwise the $\mathrm{C}$ first in the list of query in $1 \mathrm{HL}$ to record $(W, h)$, and then randomly selected $\bar{r} \in Z_{p}^{*}, \quad$ answer $\quad \bar{D}=(\bar{G}, \bar{t}), \quad$ where $\quad \bar{G}=\bar{r} p$, $\bar{t}=(x \bar{e}+\bar{r}) \bmod p, \bar{e}=h \bmod p$, and the $(W, \bar{D})$ is added to the $L_{1}$.

Proving Properties Simulation: let $n_{A}$ be $A$ nonce produced by the attacker, the attacker chooses $\left(n_{u}, W\right)$ request proved to possess the attribute set to $W$ before $W$, if asked Oracle Attest, it returns the same result, otherwise the C first in the list of query in IL to record $(W, \bar{D})$, and then randomly selected $\bar{R} \in Z_{P}^{*}, \quad P_{C}=\bar{S} P_{1}$ calculation, $\bar{m}=$ $H_{1}\left(n_{A}, W, P_{C}\right), \quad \bar{Q}=\bar{r}^{\prime}(P+\bar{G})(\bar{Q} \neq \bar{G}), \quad \overline{\sigma_{1}}=\left(\bar{m}+X_{\bar{Q}}\right)$ $\bmod p, \quad \overline{\sigma_{2}}=\left(\bar{r}-\bar{t} \overline{\sigma_{1}}\right) \bmod p$ answer property signature $\left.\bar{T}=\left(\overline{\sigma_{1}}+\bar{r}^{\prime}\right) \bar{G} \quad \overline{\sigma=\left(\overline{\sigma_{1}}\right.} \overline{\sigma_{2}}\right) \quad$ and $\quad \bar{T}, \quad P_{C}, \quad$ and $\left(\bar{m}, W, \bar{\sigma}, \bar{T}, P_{C}\right)$ into $L_{S}$.

Assuming the $\left(m^{*}, w^{*}\right)$ without being asked, if the attacker $A$ in the choice of message $m^{*}$ and choose Properties under $w^{*}$ attack with non-negligible probability of successfully forged a property signature we can assume that A signed in before forged attribute query $q_{s}$ Oracle Attest, the SQ is the name of a query answer to sign 
$\sigma^{(i)}=\left(\sigma_{1}^{(i)}, \sigma_{2}{ }^{(i)}\right) \neq\left(\sigma_{1}^{*}, \sigma_{2}{ }^{*}\right)\left(i=1,2, \ldots, q_{s}\right)$, the following two approaches to discuss properties of signature unforgeability.

1) For the $\sigma^{(i)}=\left(\sigma_{1}^{(i)}, \sigma_{2}{ }^{(i)}\right)\left(i=1,2, \ldots, q_{s}\right)$, at least in the presence of $\varepsilon / 2$ probability satisfy $\sigma_{2}{ }^{*}+x \sigma_{1}{ }^{*}=\sigma_{2}{ }^{(i)}+x \sigma_{1}{ }^{(i)}$. Calculates the $\mathrm{A}$ in this case $x=\left(\left(\sigma_{2}{ }^{(i)}-\sigma_{2}{ }^{*}\right) /\left(\sigma_{1}{ }^{*}-\sigma_{1}{ }^{(i)}\right)\right) \bmod p$ so $\mathrm{C}$ can use $\mathrm{A}$ to solve the DLP.

2) For the $\sigma^{(i)}=\left(\sigma_{1}^{(i)}, \sigma_{2}{ }^{(i)}\right)\left(i=1,2, \ldots, q_{s}\right)$, at least by $\varepsilon / 2$ probability satisfy $\sigma_{2}{ }^{*}+x \sigma_{1}{ }^{*} \neq \sigma_{2}{ }^{(i)}+x \sigma_{1}{ }^{(i)}$. At this point, according to verify the equality (1): $\left[\sigma_{2}{ }^{*} P+e^{*} \sigma_{1}^{*} P_{T C}+T^{*}\right]_{x}=\left[\sigma_{2}^{*} P+e^{*} \sigma_{1}^{*} x P+\sigma_{1}^{*} r^{*} P+r^{* *} r^{*} P\right]_{x}=\left[r^{* *} P+r^{* *} r^{*} P\right]_{x}$ professional $T^{*}=\left(\sigma_{1}^{*}+r^{* *}\right) G^{*}, G^{*}=r^{*} P, \quad e^{*}=H_{1}\left(W^{*}\right)$, $r^{*}, r^{\prime *} \in Z_{p}^{*}$ two random number'pr is produced in the process of $A$ forging. So $A$ Computing: $x=\left(\left(r^{* *}-\sigma_{2}{ }^{*}-\sigma_{1}{ }^{*} r^{*}\right) /\left(\sigma_{1}^{*} e^{*}\right)\right) \bmod p$, so $\mathrm{C}$ can also use $\mathrm{A}$ to solve the DLP.

In short, if the attacker $A$ can with non negligible probability $\mathcal{E}$ of forging property signature $\sigma^{*}$, the probability of existence of $C$ attack algorithm can use the $A$ to solve the DLP can not be ignored.

Theorem (3) RAA-TCCP protocol with anonymity, platform collusion resistance and configuration of privacy protection.

Proof: This article built on RAA-TCCP protocol based on the attributes of a ring signed on, due to the function $F: G P C R V A L S \rightarrow P R O P V A L S$ is a many to many mapping, $l=\left|\left\{V_{P C R-X} \mid V_{P C R-X} \in G P C R V A L S, F\left(V_{P C R-X}\right)=\{\omega\}_{1 \leq i \leq n} \subseteq P R O P V A L S\right\}\right| \geq 1$, and 1 is usually the number of computing nodes with $\{\omega\}_{1 \leq i \leq n}$, so the adversary guess probability ring outside the real signer's identity is not more than $1 / l$, the probability of ring inside adversary guess the real signer's identity is not more than $1 /(l-1)$, that is, the RAA-TCCP protocol has platform identity anonymity.

In addition, although the RAA-TCCP protocol in attribute certification requirements of $\mathrm{N}$ provide $\mathrm{EK}$ certificate and platform configuration information to the TC, but the agreement prove to $\mathrm{U}$ in N,N only provides property signature information $\sigma$ and $\mathrm{T}$, TC even with signature key $D=(G, t)$ of $\mathrm{N}$, cannot be calculated $\sigma$ and $\mathrm{T}$, which makes $\mathrm{N}$ are anonymous to $\mathrm{U}$ and $\mathrm{TC}$, so $\mathrm{U}$ cannot TC N and conspiracy to get the true identity of. At the same time, TC also can't and $\mathrm{N}$ conspiracy to deceive the $\mathrm{U}$, because the signature key can only be issued by TC generating property legal signtheature, and the sinatuer key by binding and sealing cannot be migrated to other nodes using $\mathrm{TC}$, this cannot be denied. Terefore, the RAA-TCCP protocol has the collusion resistance. Because of this platform identity anonymity and collusion resistance, the platform configuration information provided to $\mathrm{TC} \mathrm{N}$ will also not be exposed, therefore, the RAA-TCCP protocol has the configuration privacy protection.

\subsection{Validity}

RAA-TCCP protocol is a simple, effective. Firstly, the RAA-TCCP protocol and realizes the identity and integrity of the state of computing nodes to prove. In the proof of the preparatory phase, EK certificate, certificate of conformance certificate platform and platform identity, SML and group PCR value platform integrity attestation state. While only verify the success to the corresponding security attributes and signature key, and sealing, binding to the signature key. To ensure that such implementation stage in the proof, only the holder of the signature key and the corresponding security configuration can generate legitimate property signature. The $U$ attribute can be verified by the legitimacy of the signature to judge the credibility of $\mathrm{N}$. When the user virtual machine migration occurs, the target host only be verified as authentic to obtain the session key, which can generate a valid HMAC, the source host according to the user virtual machine encryption to the target host, effectively ensure the confidentiality and user controllable virtual machine running.

Secondly, greatly enhance the efficiency of remote attestation. Adopting off-line trusted third party, the communication cost is low, the elimination of the efficiency bottleneck caused by using the online form; ring signature based on the attributes, so that the number of ring signature length and ring members independently, be fixed length of ring signature, eliminates the efficiency bottleneck number of ring members brought in to maintain anonymity, but also improve the efficiency of. In particular, the RAA-TCCP protocol to construct the whole scheme based on elliptic curve discrete logarithm problem, because the elliptic curve scalar multiplication cost far less than the bilinear pairing, thus greatly improve computational efficiency, than the proof protocol based on bilinear pairing at the same time, there are strict requirements on the bilinear nature of the elliptic curve, and its computational efficiency in high security intensity (1024-bit RSA the above security strength) will cause a series of problems [6]. Therefore, prove that the protocol without pairing will have lower computational complexity and higher universality. In order to efficient RAA-TCCP protocol description, in the 1024-bit RSA the same security level, the RAA-TCCP protocol and the existing typical based on bilinear pairing calculation comparison on the amount in the certification and verification proved that the scheme of PBABM [7] and AA-ABRS [8]. The PBA-BM scheme and AAABRS scheme of bilinear pairings and safety parameter selection based on literature [9] the method chooses the most efficient (160-bit r, 512-bit q) Type A curve, RAA-TCCP select 160-bit protocol stochastic elliptic curve. This paper only considers the time-consuming three operating larger: the pairing operations, scalar multiplication and exponentiation, while the PBA-BM scheme and AA-ABRS scheme known for advance computing bilinear. 
Table. 1. Comparison of calculation amount with PBA-BM, AA-ABRS scheme.

\begin{tabular}{|c|c|c|c|}
\hline Scheme & The Length of the Signature /bits & Prove the Amount of Calculation & Verify the Amount of Calculation \\
\hline PBA-BM & 5061 & ${ }_{0} T_{a+7} T_{b+12} T_{c}$ & ${ }_{4} T_{a+4} T_{b+7} T_{c}$ \\
\hline AA-ABRS & 1026 & ${ }_{0} T_{a+5} T_{b+0} T_{c}$ & ${ }_{2} T_{a+0} T_{b}+T_{c}$ \\
\hline RAA-CCP & 320 & ${ }_{0} T_{a+2} T_{b+0} T_{c}$ & ${ }_{0} T_{a+3} T_{b+0} T_{c}$ \\
\hline
\end{tabular}

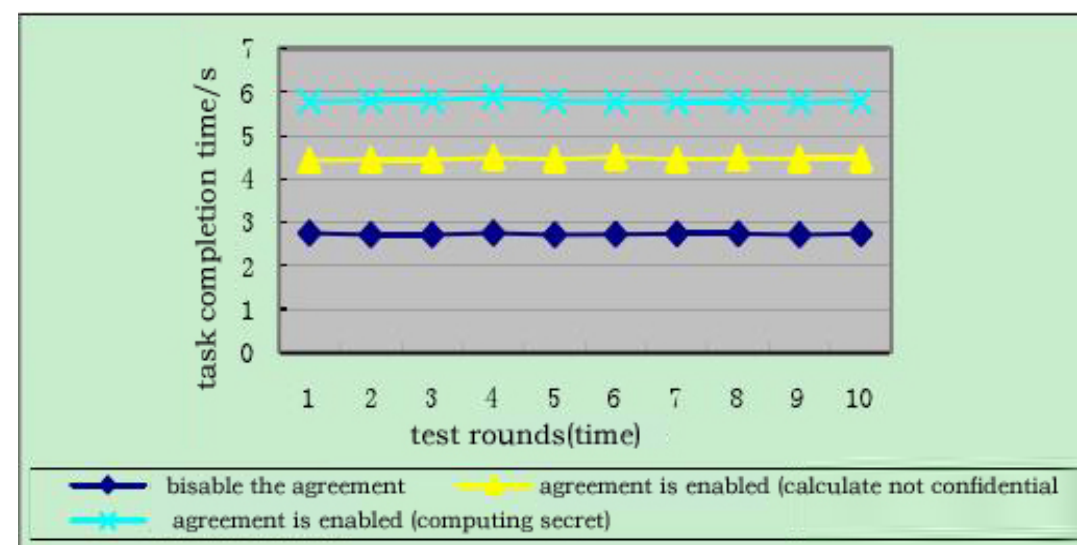

Fig. (4). RAA-TCCP protocol for computing the performance test results in the platform in the cloud.

Wherein $T_{a}$ represents a bilinear pairing operation time, $T_{b}$ represents a scalar multiplication time, $T_{c}$ said one exponentiation time. It is seen from Table 1, compared with the PBA-BM scheme of the new scheme, property signature length decreased from 5061 bits to 320 bits, 14.82 times shorter, certification and verification process is no longer a bilinear pairings and exponentiation, certification and verification efficiency has been greatly improved. At the same time, the new scheme without trusted third party issued by attribute certificate, attribute is judged through the ring signature be bound and sealed within the TPM signature key generated, so there is no attribute revocation checking problem; while the PBA-BM scheme also need to rely on an online trusted third party attribute revocation check, which increased the system management difficulty, also reduces the efficiency of the system. In addition, the new scheme in collusion resistance and no AIK certificate have unique advantages, the above characteristics make the new scheme in cloud computing has very good application platform. While the AA-ABRS scheme while also has the new scheme has the platform identity anonymity, configuration of privacy protection, collusion resistance and no attribute certificate and AIK certificate and other advantages, but because it still has 2 pairing and 1 exponentiation, so the operating efficiency of the new scheme is still high, and the properties of signature a new scheme of short length than.

\section{EXPERIMENT RESULTS AND ANALYSIS}

In order to verify the efficiency of the RAA-TCCP protocol, with a case of cloud computing based on Hadoop platform, a proof of the RAA-TCCP protocol implementation process simulation experiment. On the platform with MapReduce mode of 2218 -bit random integer (total 2MB) performs a counting task, counting using heap sort method, in order to avoid the difference of different random sequence sorting efficiency. The platform consists of 5 Pentium 4 CPU 2.0GHz, 512M memory, and are connected by a $100 \mathrm{Mbps}$ Ethernet computer built, of which 1 stations for the client, 1 to $\mathrm{CM}$, the remaining 3 Taiwan as computing nodes. The $\mathrm{CM}$ is set to master, the calculation of the node is set to slaves, the 3 slaves is master assigned tasks are proved. Due to the current real TPM does not support the protocol of elliptic curve cryptography, this paper uses BouncyCastle library, use NIST 256-bit elliptic curve, RIPEMD160 hash, 128-bitAES symmetric cryptography, is the time to complete the task in the enabled RAA-TCCP co disable RAA-TCCP protocol, protocol (Computing unclassified), enable RAATCCP protocol (computing the confidential) for such as shown in Fig. (4). At the same time, in the same security level, single proof and verification process of the RAATCCP protocol, the PBA-BM scheme and AA-ABRS scheme also made analogy experiment. The experiment, in order to avoid the bilinear pairing initiated in high security problems in the strength, 160-bit $r$ select the PBA-BM scheme and AA-ABRS scheme, the 512-bit $q$ TypeA curve, the jPBC Library (Java Pairing-based Cryptography Library) [10] implementation; and the RAA-TCCP protocol, the choice of 160-bit random elliptic curve, the performance of the control as shown in Fig. (5).

Experimental results show that the RAA-TCCP protocol has high operation efficiency of computing platform in the cloud. In the 3072-bit RSA security strength, for counting task 221 integer 8-bit, in the calculation of confidential, the average task completion time is $4.454 \mathrm{~s}$; in 


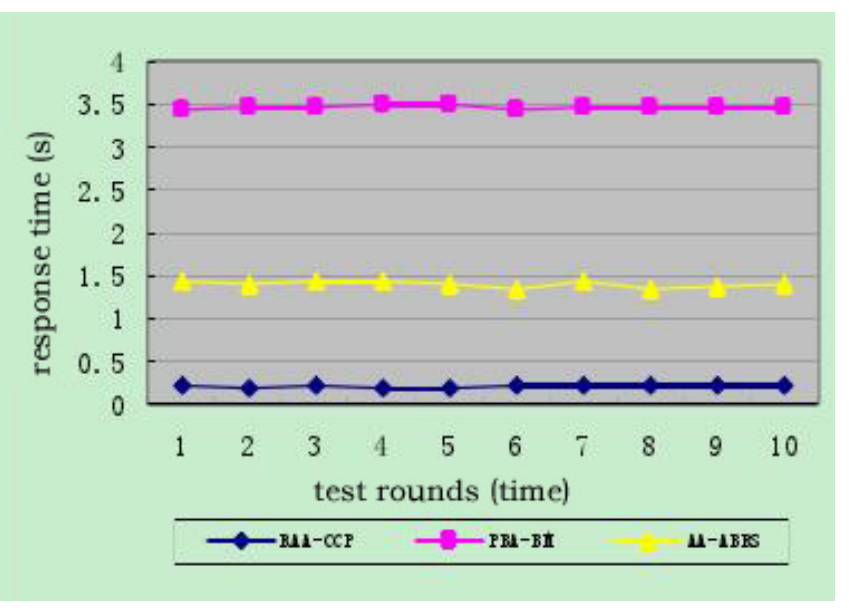

Fig. (5). RAA-TCCP and PBA-BM, the performance of AA-ABRS.

The calculation of secrecy, the average task completion time is $5.787 \mathrm{~s}$; and when to disable the RAA-TCCP protocol, the average task completion time for $2.721 \mathrm{~s}$, delay RAA-TCCP protocol brought in calculation is not confidential and keep were 4.454-2.721=1.734s and 5.787$2.721=3.066 \mathrm{~s}$. But in the process, the RAA-TCCP protocol is executed 3 times, computational secrecy, $1 \mathrm{MB}$ task 128bitAES of data encryption - decryption executed 4 times, therefore, a delay in bringing single RAA-TCCP protocol process average of only $1.734 / 3=0.578 \mathrm{~s}$, delayed $0.578 / 2.721 * 100 \%=21.24 \%$; delay of single $1 \mathrm{MB}$ mission data encryption or decryption brought only an average of $(3.066-1.734) /(2 * 4) \quad=0.167 \mathrm{~s}, \quad$ delayed $0.167 / 2.721 *$ $100 \%=6.14 \%$. Comparison of PBA-BM and AA-ABRS in the 1024-bitRSA scheme, RAA-TCCP protocol security strength, the average response time for $0.207 \mathrm{~s}$, PBA-BM average response time is $3.467 \mathrm{~s}$ (excluding attribute revocation check time), the average response time for the $1.407 \mathrm{~s}$ AA-ABRS scheme, the new scheme is 15.76 times faster than the PBA-BM scheme, AA-ABRS scheme is 5.80 times faster than. The above performance is mainly because the new scheme is no longer a bilinear pairings and exponentia- tion, therefore, even in a high security level, also has the very high operating efficiency.

\section{CONFLICT OF INTEREST}

The authors confirm that this article content has no conflict of interest.

\section{ACKNOWLEDGEMENTS}

This work was financially supported by the Henan Science and Technology Key Project Foundation (122102210507).

\section{ABOUT THE AUTHORS}

First Author Liu Yan, University lecturer of Zhongzhou University, Master. The author's major is Information Security.

Second Author Xia Bin, University lecturer of Henan Agricultural University, Master. The author's major is Data mining.

\section{REFERENCES}

[1] T. C. P. Alliance, "TCPA design philosophies and concepts (version 1)," https://www.trustedcomputinggroup.org, 2001.

[2] J. Liu, J. Zhao, and Y. Zhao, "Remote automatic anonymous attestation in trusted computing," Chinese Journal of Computers, vol. 7, pp. 1304-1310, 2009.

[3] L.Q. Chen, and H. Löhr, and M. Manulis, "Property-based attestation without a trusted third party," Proceedings of $11^{\text {th }}$ International Conference on ICS, Taiwan, pp.31-46, 2008.

[4] N. Santos, and K.P. Gummadi, and R. Rodrigues, Towards Trusted Cloud Computing: CA, Berkeley, pp.56-68, 2009.

[5] W. Q. Wang and S. Z. Chen, An Efficient Attribute-Based Ring Signature Scheme: CA, Piscataway, pp.147-150, 2009.

[6] L. Hu, "Compression of Tate pairings on elliptic curves", Journal of Software, vol. 18, pp. 1799-1805, 2007.

[7] D. G. Feng, and Y. Qin, "A property-based attestation protocol for TCM," Sciences China, vol. 53, no. 3, pp. 454-464, 2010.

[8] D. J. Luo, and J. Zhang, "An efficient anonymous attestation attribute ring signature based on the property ring signature anonymity prove efficient protocol," Computer Application Research, vol 12, no. 5, pp. 3470-3474, 2012.

[9] B. Lynn, On the Implementation of Pairing-Based Cryptosystems, Stanford University, California, pp. 420-441, 2007.

[10] B. Lynn, "The pairing-based cryptography library," http://crypto. stanford.edu/pbc/times.html, 2006.

(C) Yan and Bin; Licensee Bentham Open.

This is an open access article licensed under the terms of the Creative Commons Attribution Non-Commercial License (http://creativecommons.org/licenses/by-nc/3.0/) which permits unrestricted, non-commercial use, distribution and reproduction in any medium, provided the work is properly cited. 